\title{
Consensus of Second-order Discrete Multi-agent System with Communication Delay and Switching Topology
}

\author{
Kexin Wang ${ }^{1+}$, Jing Zhang ${ }^{2}$ \\ ${ }^{1}$ School of Electronic and Information Engineering, Beijing Jiaotong University, Beijing, China,100044
}

\begin{abstract}
This paper studies the conditions of consensus of a multi-agent system under distributed network when communication delay and switching topology existing. The delay we considered in this paper is caused by the distributed network protocol instead of the process of transmission. The switching of topology is caused by the instability of the network. We first construct relevant system model where communication delay and switching topology both exist. And then we obtain the sufficient conditions to guarantee that a set of agents reach consensus on their states, including positions and velocities. Finally, numerical simulations are provided to illustrate the availability of our results. The main contribution of our research is that we consider the delay under distributed network protocol, which makes the results more practical and can be used as a guide in both the design of controller and the optimizing of the network protocol for consensus of the system under distributed network.
\end{abstract}

Keywords: Consensus, Communication Delay, Switching Topology, Distributed Network.

\section{Introduction}

In recent years, consensus of distributed multi-agent system has attracted a number of researchers to study. This kind of upsurge is mainly caused by extensive applications of relevant theory in many fields including flocking [1]-[2], formation control [3]-[4] and swarming [5], etc.

A number of researchers have analyzed the multi-agent system described by single-order integrators [6][8]. It is shown in [7] that if the time-invariant topology of the communication network has a spanning tree, the multi-agent system under this kind of topology can finally reach consensus by choosing appropriate parameter. As relevant research moves along, scholars pay more attention on second-order discrete multiagent system, which is more grounded in reality. [9] studies on the relationship between the control gain and the sampling period when consensus of a sampled-data double-integrator system is reached.

During the process of interaction, communication constraints are inevitable. [10]-[11] analyze the system with communication delay, they show the sufficient conditions for reaching consensus and the way of controller designing by using Lyapunov function. [12] studies on the system with link failures and channel noise and finds the influence of those imperfect communication factors and present two compromises. [13] analyzes the system with noise including not only systematic but also measured one. The author used Kalman filtering to design the controller to recede the influence of noise. The results mentioned above consider the system with some communication constraints. However, there is little research focusing on the communication delay caused by the distributed network protocol, which is more realistic and more useful for both the designation of controller and optimization of the network.

In this paper, we focus on if the agents in a second-order discrete system could reach expected values on their states including positions and velocities when communication delay and topological switching both exist in the system. We construct relevant models and obtain the sufficient conditions to ensure the

\footnotetext{
+ Corresponding author. Tel.: +86-15801087882

E-mail address: 18120135@bjtu.edu.cn
} 
consensus of the agents under our distributed network. Our result shows that the adjustment of control parameters and the optimizing of the network protocol are both effective methods to guarantee the achievement of consensus.

\section{Backgrounds Statements}

\subsection{Graph and Topology Theory}

Graphs are usually used to describe the communication amongst $N$ agents. Considering $\mathcal{G} \triangleq(\mathcal{V}, \mathcal{E}, \mathcal{A})$ is an undirected graph consists of the node set $\mathcal{V} \triangleq\{1,2, \cdots, N\}$, the edge set $\mathcal{E} \subseteq \mathcal{V} \times \mathcal{V}$ and the weighted adjacency matrix $\mathcal{A}=\left[a_{i j}\right] \in \mathbb{R}^{N \times N}$. Since $\mathcal{G}$ is an undirected graph, an edge $(i, j) \in \mathcal{E}$ denotes that agent $i$ and $j$ are able to get information from each other. $\mathcal{A}$ is defined as $a_{i j}>0$ if $(i, j) \in \mathcal{E}$, while $a_{i j}=0$ if $(i, j) \notin \mathcal{E}, a_{i i}=0$. In this paper, we suppose that $a_{i j}=1$ if $(i, j) \in \mathcal{E}$. Laplacian matrix $\mathcal{L}=\left[\ell_{i j}\right] \in \mathbb{R}^{N \times N}$ is defined as $\ell_{i i}=\sum_{j=1, i \neq j}^{N} a_{i j}$, if $i \neq j, \ell_{i j}=-a_{i j}$. In this paper the multi-agent system has $N$ following agents and one leading agent, so matrix $\Omega=\left[\omega_{i j}\right] \in \mathbb{R}^{N \times N}$ is defined as $\omega_{i i}=1$ if the following agent $i$ can obtain the information of the leading agent and $\omega_{i j}=0$ if $i \neq j$. For the matrix $\Omega+\mathcal{L}$, we have

Lemma 2.1. If the topology consists of $N$ followers is connected and there is at least one of following agent having the access to obtain the data of leading agent, all the eigenvalues of matrix $\Omega+\mathcal{L}$ are positive.

\subsection{Second-order Discrete Multi-agent System}

The dynamic equations of the follower $i$ and the leading agent are given by

$$
\begin{gathered}
p_{i}[k+1]=p_{i}[k]+h v_{i}[k]+\frac{h^{2}}{2} u_{i}[k], v_{i}[k+1]=v_{i}[k]+h u_{i}[k] \\
p_{L}[k+1]=p_{L}[k]+h v_{L}[k], v_{L}[k+1]=v_{L}[k]
\end{gathered}
$$

where $h$ is the sampling interval of the system and $k$ represents the index of discrete time. The position and velocity of the follower $i$ at $t=k h$ are represented by $p_{i}[k] \in \mathbb{R}^{p}$ and $v_{i}[k] \in \mathbb{R}^{p}$, while the same states of leader at $t=k h$ are denoted by $p_{L}[k] \in \mathbb{R}^{p}$ and $v_{L}[k] \in \mathbb{R}^{p} . u_{i}[k] \in \mathbb{R}^{p}$ which has the physical meaning of the acceleration, represents control input of the following agent $i$ at $t=k h$. In this paper we assume that $\mathrm{p}=1$. The control input $u_{i}[k]$ takes the form of

$$
\begin{array}{r}
u_{i}[k]=\omega_{i i} \beta\left[\left(p_{L}[k]-p_{i}[k]\right)+\gamma\left(v_{L}[k]-v_{i}[k]\right)\right]+ \\
\beta \sum_{j \in N_{i}} a_{i j}\left[\left(p_{j}[k]-p_{i}[k]\right)+\gamma\left(v_{j}[k]-v_{i}[k]\right)\right]
\end{array}
$$

where $\beta$ and $\gamma$ are two positive numbers introducing input gain and relative damping. $\omega_{i i}$ and $a_{i j}$ have the same definition as the parameters in section 2.1.

Since the target is the consensus of the followers, which means that they reach expected states with the leading agent, we have the following error system

$$
\begin{aligned}
& p_{i}[k+1]-p_{L}[k+1]=p_{i}[k]-p_{L}[k]+h\left(v_{i}[k]-v_{L}[k]\right)+\frac{h^{2}}{2} u_{i}[k] \\
& v_{i}[k+1]-v_{L}[k+1]=v_{i}[k]-v_{L}[k]+h u_{i}[k]
\end{aligned}
$$

\subsection{Communication Delay Model Based on Network Protocol}

The delay is caused by allocation of communication resources under specific protocols. We focus on MAC protocols of distributed network and Fig. 1 shows that the communication resources are described by the frame, which consists of control subframe and data subframe. DSCH messages in control slots are used to reserve the communication resources. To avoid the collision, the protocol has an election strategy. For the nodes in the network, there is expected interval between two times competing for the control slots. Since we put the state of agents using for the calculating of the control input into DSCH message, the communication delay $h$ could be denoted by the expected interval. The interval is related to the network parameters including back-off exponent, the number of two-hoops neighbor, etc. This relation gives us the direction to optimize the protocol base on our results. 


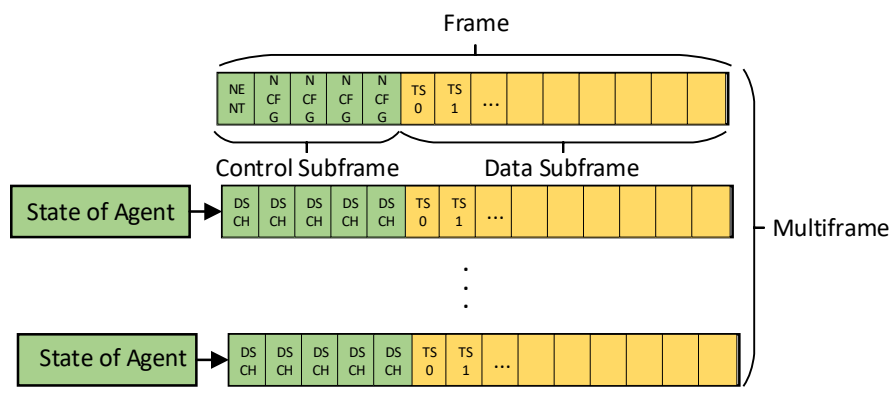

Fig. 1: The structure of frame in distributed network.

\section{Consensus Analysis of the System under Communication Delay and Switching Topology}

In this subsection, we analyze the conditions to ensure the consensus under communication delay and switching topology. Considering that communication delay and switching topology exists when agents exchange their information, the control input (3) has the following form

$$
\begin{aligned}
& u_{i}[k]=\omega_{i i}\left[r_{k-1}\right] \beta\left[\left(p_{L}[k-1]-p_{i}[k-1]\right)+\gamma\left(v_{L}[k-1]-v_{i}[k-1]\right)\right] \\
& +\beta \sum_{j \in N_{i}} a_{i j}\left[r_{k-1}\right]\left[\left(p_{j}[k-1]-p_{i}[k-1]\right)+\gamma\left(v_{j}[k-1]-v_{i}[k-1]\right)\right]
\end{aligned}
$$

The input at $t=k h$ is calculate base on the data at $t=(k-1) h$, so the communication delay is $1 \times h$. In this paper the sampling period and communication delay are the same parameter $h$. And $r_{k-1}=i, 1 \leqslant i \leqslant M$ denotes that the topology at $t=(k-1) h$ is the $i t h$ pattern. The total number of patterns is M. Using (5) for (1) and (2) indicates that the multi-agent system has inevitable communication delay and the topology is switchover. The matrix $\Omega+\mathcal{L}$ in section 2.1 turns to $\Omega\left[r_{k}\right]+\mathcal{L}\left[r_{k}\right]$.We define $\Lambda\left[r_{k}\right]=\beta\left(\Omega\left[r_{k}\right]+\mathcal{L}\left[r_{k}\right]\right)$.

The sufficient conditions when consensus of system (1) and (2) are reached under the control law (5) can be stated in Theorem 3.1 .

Theorem 3.1. Consensus of system (1) and (2) can be reached under control law (5) if lemma 2.1 and the following inequation are satisfied

$$
h<\min \left\{\gamma-\sqrt{\gamma^{2}-\frac{2}{\mu_{\max }}}, \frac{4\left(\gamma \mu_{\max }-\left(\sqrt[3]{Y_{1}}+\sqrt[3]{Y_{2}}\right)\right)}{3 \mu_{\max }}\right\}
$$

where $Y_{i}=\frac{1}{8} \gamma^{3} \mu_{\max }^{3}+\frac{27}{32} \gamma \mu_{\max }^{2} \pm \frac{3}{8} \mu_{\max } \sqrt{3 \gamma^{4} \mu_{\max }^{3}-\frac{27}{16} \gamma^{2} \mu_{\max }^{2}+\frac{81}{8} \mu_{\max }}, i=1,2, \mu_{\max }$ is the maximum eigenvalue of matrix $\Lambda\left[r_{k}\right]$.

Before our proof, we need to introduce some lemmas, including:

Lemma 3.1. Let $A_{1}, A_{2}, A_{3}$ and $A_{4} \in R^{n \times n}$. Let $M=\left[\begin{array}{ll}A_{1} & A_{2} \\ A_{3} & A_{4}\end{array}\right]$, the determinant of matrix $M$ : $\operatorname{det}(M)=\operatorname{det}\left(A_{1} A_{4}-A_{2} A_{3}\right)$.

Lemma 3.2 (Julie's criterion). For the equation $s^{3}+a s^{2}+b s+c=0$, all modulus of the roots are less than one if and only if the following conditions are satisfied simultaneously: $a+b+c+1>0, a-b+c-1<0,|c|<1$, $\left|c^{2}-1\right|>|a c-b|$.

Lemma 3.3. If all modulus of the eigenvalues of $M \in \mathbb{R}^{n \times n}$ are less than one, there must be a matrix norm $\|\cdot\|_{*}$ satisfying $\|M\|_{*}<1$.

Proof of Theorem 3.1. Since system (4) is equivalent to system (1) and system (2), our analyzation base on (4). Substituting formula (5) into (4), the matrix form of (4) can be denoted as

$$
\underbrace{\left[\begin{array}{c}
p[k+1]-p_{L}[k+1] \\
v[k+1]-v_{L}[k+1] \\
p[k]-p_{L}[k] \\
v[k]-v_{L}[k]
\end{array}\right]}_{\delta[k+1]}=\underbrace{\left[\begin{array}{cccc}
I_{N} & h I_{N} & -\frac{h^{2}}{2} \Lambda\left[r_{k}\right] & -\frac{h^{2}}{2} \gamma \Lambda\left[r_{k}\right] \\
0 & I_{N} & -h \Lambda\left[r_{k}\right] & -h \gamma \Lambda\left[r_{k}\right] \\
I_{N} & 0 & 0 & 0 \\
0 & I_{N} & 0 & 0
\end{array}\right]}_{F\left[r_{k}\right]} \underbrace{\left[\begin{array}{c}
p[k]-p_{L}[k] \\
v[k]-v_{L}[k] \\
p[k-1]-p_{L}[k-1] \\
v[k-1]-v_{L}[k-1]
\end{array}\right]}_{\delta[k]}
$$


The parameter $p[k]=\left[p_{1}[k], p_{2}[k], \cdots, p_{N}[k]\right]^{T}, v[k]=\left[v_{1}[k], v_{2}[k], \cdots, v_{N}[k]\right]^{T}$, $p_{L}[k]=\left[p_{L}[k], p_{L}[k], \cdots, p_{L}[k]\right]_{1 \times N}^{T}, v_{L}[k]=\left[v_{L}[k], v_{L}[k], \cdots, v_{L}[k]\right]_{1 \times N}^{T}$, and $I_{N}$ is an $N \times N$ identity matrix. With the iteration, (7) can be written as

$$
\delta[k+1]=\prod_{n=1}^{k} F\left[r_{n}\right] \delta[0]
$$

where $\delta[0]$ represent the initial difference of status between leader and followers.

The multi-agent systems reach consensus when the following equation is satisfied

$$
\lim _{k \rightarrow \infty}\|E(\delta[k])\|_{2}=0
$$

Suppose that the switching of topology is an independent process and $\Lambda\left[r_{k}\right]$ is identically distributed, then we have

$$
\begin{aligned}
\lim _{k \rightarrow \infty}\|E(\delta[k+1])\|_{2} & \leqslant \lim _{k \rightarrow \infty}\left\|\prod_{n=1}^{k} E\left(F\left[r_{n}\right]\right)\right\|_{2}\|\delta[0]\|_{2} \\
& =\lim _{k \rightarrow \infty}\left\|\bar{F}^{k}\right\|_{2}\|\delta[0]\|_{2}
\end{aligned}
$$

$\Lambda\left[r_{k}\right]$ turns to $\bar{\Lambda}=E\left(\Lambda\left[r_{k}\right]\right)$ in $\bar{F}$. Suppose that $\bar{\mu}_{i}$ is the $i$ th eigenvalue of $\bar{\Lambda}$.

We concentrate on the eigenvalues of $F\left[r_{k}\right]$. According to lemma 3.1 mentioned above, the characteristic polynomial of matrix $F\left[r_{k}\right]$ could be calculated by the following equation

$$
\begin{aligned}
& \operatorname{det}\left(s I_{4 N}-F\left[r_{k}\right]\right) \\
= & \operatorname{det}\left(\left[\begin{array}{cc}
s(s-1) I_{N}+\frac{h^{2}}{2} \Lambda\left[r_{k}\right] & -h s I_{N}+\frac{h^{2}}{2} \gamma \Lambda\left[r_{k}\right] \\
h \Lambda\left[r_{k}\right] & s(s-1) I_{N}+h \gamma \Lambda\left[r_{k}\right]
\end{array}\right]\right) \\
= & \operatorname{det}\left(s^{2}(s-1)^{2} I_{N}+\frac{h^{2}}{2} \Lambda\left[r_{k}\right] s(s-1)+h \gamma \Lambda\left[r_{k}\right] s(s-1)+h^{2} \Lambda\left[r_{k}\right] s\right) \\
= & s^{N} \operatorname{det}\left(s(s-1)^{2} I_{N}+\left(\frac{h^{2}}{2}(s-1)+h \gamma(s-1)+h^{2}\right) \Lambda\left[r_{k}\right]\right)
\end{aligned}
$$

Let $\mu_{i_{k}}$ be the ith eigenvalue of matrix $\Lambda\left[r_{k}\right]$, (11) can be written as

$$
\prod_{i=1}^{N}\left(s^{3}-2 s^{2}+\left(\frac{h^{2}}{2} \mu_{i r_{k}}+h \gamma \mu_{i r_{k}}+1\right) s+\left(\frac{h^{2}}{2}-h \gamma\right) \mu_{i n_{k}}\right)=0
$$

Obviously (12) satisfy the following equation

$$
s^{3}-2 s^{2}+\left(\frac{h^{2}}{2} \mu_{i r_{k}}+h \gamma \mu_{i r_{k}}+1\right) s+\left(\frac{h^{2}}{2}-h \gamma\right) \mu_{i r_{k}}=0
$$

Matrix $F\left[r_{k}\right]$ has $4 \times N$ eigenvalues, a quarter of them are zero, and the rest are determined by eigenvalues $\mu_{i n_{k}}(i=1,2, \cdots, N)$, each $\mu_{i n_{k}}$ corresponds to three eigenvalues of $F\left[r_{k}\right]$. We suppose that topology consists of $N$ followers is connected and there is at least one of following agent having the access to obtain the information of leading agent in every pattern of the topology. From lemma 2.1, we know that $\mu_{i r_{\mathrm{k}}}>0$. Using lemma 3.2, $|s|<1$ is satisfied when the following inequations are satisfied simultaneously

$$
\begin{gathered}
-2+\left(\frac{h^{2}}{2} \mu_{i r_{k}}+h \gamma \mu_{i r_{k}}+1\right)+\left(\frac{h^{2}}{2}-h \gamma\right) \mu_{i r_{k}}+1>0 \\
-2-\left(\frac{h^{2}}{2} \mu_{i r_{k}}+h \gamma \mu_{i r_{k}}+1\right)+\left(\frac{h^{2}}{2}-h \gamma\right) \mu_{i r_{k}}-1<0 \\
\left|\left(\frac{h^{2}}{2}-h \gamma\right) \mu_{i r_{k}}\right|<1 \\
\left|\left(\left(\frac{h^{2}}{2}-h \gamma\right) \mu_{i r_{k}}\right)^{2}-1\right|>\left|-2\left(\frac{h^{2}}{2}-h \gamma\right) \mu_{i r_{k}}-\left(\frac{h^{2}}{2} \mu_{i r_{k}}+h \gamma \mu_{i r_{k}}+1\right)\right|
\end{gathered}
$$

Obviously (14) and (15) are true. (16) can be simplified as the following inequation

$$
h<\gamma-\sqrt{\gamma^{2}-\frac{2}{\mu_{\max }}}
$$


where $h$ denotes the communication delay, $\gamma$ denotes the relative damping and $\mu_{\max }=\max \left\{\mu_{i r_{k}}\right\}$ is the maximum eigenvalue of matrix $\Lambda\left[r_{k}\right]$.

The inequation (17) can be simplified as

$$
\frac{\mu_{i r_{k}}}{4} h^{3}-\gamma \mu_{i r_{k}} h^{2}+\left(\gamma^{2} \mu_{i r_{k}}+\frac{3}{2}\right) h-\gamma<0
$$

Using Shengjin's Formulas in [14], (19) can be simplified as

$$
h<\frac{4\left(\gamma \mu_{\max }-\left(\sqrt[3]{Y_{1}}+\sqrt[3]{Y_{2}}\right)\right)}{3 \mu_{\max }}
$$

where $Y_{i}=\frac{1}{8} \gamma^{3} \mu_{\max }^{3}+\frac{27}{32} \gamma \mu_{\max }^{2} \pm \frac{3}{8} \mu_{\max } \sqrt{3 \gamma^{4} \mu_{\max }^{3}-\frac{27}{16} \gamma^{2} \mu_{\max }^{2}+\frac{81}{8} \mu_{\max }}, i=1,2$.

Using (18) and (20), we have

$$
h<\min \left\{\gamma-\sqrt{\gamma^{2}-\frac{2}{\mu_{\max }}}, \frac{4\left(\gamma \mu_{\max }-\left(\sqrt[3]{Y_{1}}+\sqrt[3]{Y_{2}}\right)\right)}{3 \mu_{\max }}\right\}
$$

Since $\bar{\Lambda}$ is the expected value of $\Lambda\left[r_{k}\right]$, it is obvious that $\bar{\mu}_{i}<\mu_{\max }$. This means that if (21) is satisfied, all modulus of the eigenvalues of $\bar{F}$ in inequality (10) are less than one. Using lemma 3.3 mentioned above and matrix norm equivalence, (10) can be simplified as

$$
\begin{aligned}
\lim _{k \rightarrow \infty}\|E(\delta[k+1])\|_{2} & \leqslant \lim _{k \rightarrow \infty}\left\|\bar{F}^{k}\right\|_{2}\|\delta[0]\|_{2} \\
& \leqslant \lim _{k \rightarrow \infty} C_{*}\left\|\bar{F}^{k}\right\|_{*}\|\delta[0]\|_{2} \\
& \leqslant \lim _{k \rightarrow \infty} C_{*}\|\bar{F}\|_{*}^{k}\|\delta[0]\|_{2}=0
\end{aligned}
$$

where $C_{*}$ denotes a positive const, $\|\cdot\|_{*}<1$ is a matrix norm. Using (9), system can reach consensus.

Theorem 3.1 has been proved.

\section{Simulation Results}

In this subsection, we take four numerical simulation experiments to prove the correctness of theorem 3.1. There are five following agents and one leading agent and there are four patterns of switching topology given by (23) and (24). The state of switching topology are the same in four experiments. System parameters $\beta, \gamma$ and $h$ set by us are shown in Table 1 . And the upper bound of delay $h_{\max }$ is calculated by (21).

$$
\begin{array}{r}
\Lambda\left[r_{1}\right]=\beta\left[\begin{array}{rrrrr}
2 & -1 & 0 & 0 & -1 \\
-1 & 2 & -1 & 0 & 0 \\
0 & -1 & 4 & -1 & -1 \\
0 & 0 & -1 & 3 & -1 \\
-1 & 0 & -1 & -1 & 3
\end{array}\right], \Lambda\left[r_{2}\right]=\beta\left[\begin{array}{rrrrr}
2 & -1 & 0 & 0 & -1 \\
-1 & 2 & -1 & 0 & 0 \\
0 & -1 & 3 & -1 & 0 \\
0 & 0 & -1 & 3 & -1 \\
-1 & 0 & 0 & -1 & 2
\end{array}\right] \\
\Lambda\left[r_{3}\right]=\beta\left[\begin{array}{rrrrr}
2 & -1 & 0 & 0 & -1 \\
-1 & 2 & -1 & 0 & 0 \\
0 & -1 & 4 & -1 & -1 \\
0 & 0 & -1 & 2 & -1 \\
-1 & 0 & -1 & -1 & 3
\end{array}\right], \Lambda\left[r_{4}\right]=\beta\left[\begin{array}{rrrrr}
2 & -1 & 0 & 0 & -1 \\
-1 & 2 & -1 & 0 & 0 \\
0 & -1 & 2 & -1 & 0 \\
0 & 0 & -1 & 3 & -1 \\
-1 & 0 & 0 & -1 & 2
\end{array}\right] \\
\text { Table 1: The setting of parameters in different experiments }
\end{array}
$$

\begin{tabular}{ccccc}
\hline Name & $\beta$ & $\gamma$ & $h$ & $h_{\max }$ \\
\hline experiment (a) & 1.5 & 1.2 & 0.1 & 0.1028 \\
experiment (b) & 1.1 & 1.2 & 0.1 & 0.1373 \\
experiment (c) & 0.7 & 1.2 & 0.1 & 0.2059 \\
experiment (d) & 1.5 & 1.2 & 0.15 & 0.1028 \\
\hline
\end{tabular}

Fig. 2 shows the state of each follower in different experiments and indicate if the followers could reach consensus. As is shown in Table 1(the fourth and the fifth columns) and Fig. 2, systems in experiment (a) experiment (c) can reach consensus because their communication delays $h$ are lower than the bound of delay 
$h_{\max }$, while the result in experiment (d) is opposite. The correctness of theorem 3.1 is illustrated. Base on the analysis in section 2.3, we can also optimize the distributed network protocol to decrease the communication delay $h$ to guarantee the achievement of consensus besides adjusting the control gain. Fig. 2 (a)- Fig. 2 (c) also indicate a positive correlation between the speed of reaching consensus and control gain $\beta$ when the delay is lower than the calculated bound. However, the second and the fifth columns of Table 1 show that the increase of $\beta$ will lead to the decrease of $h_{\max }$. The lower $h_{\max }$ will bring more constraints to the network protocol. It is significant to choose an appropriate $\beta$ for the system.
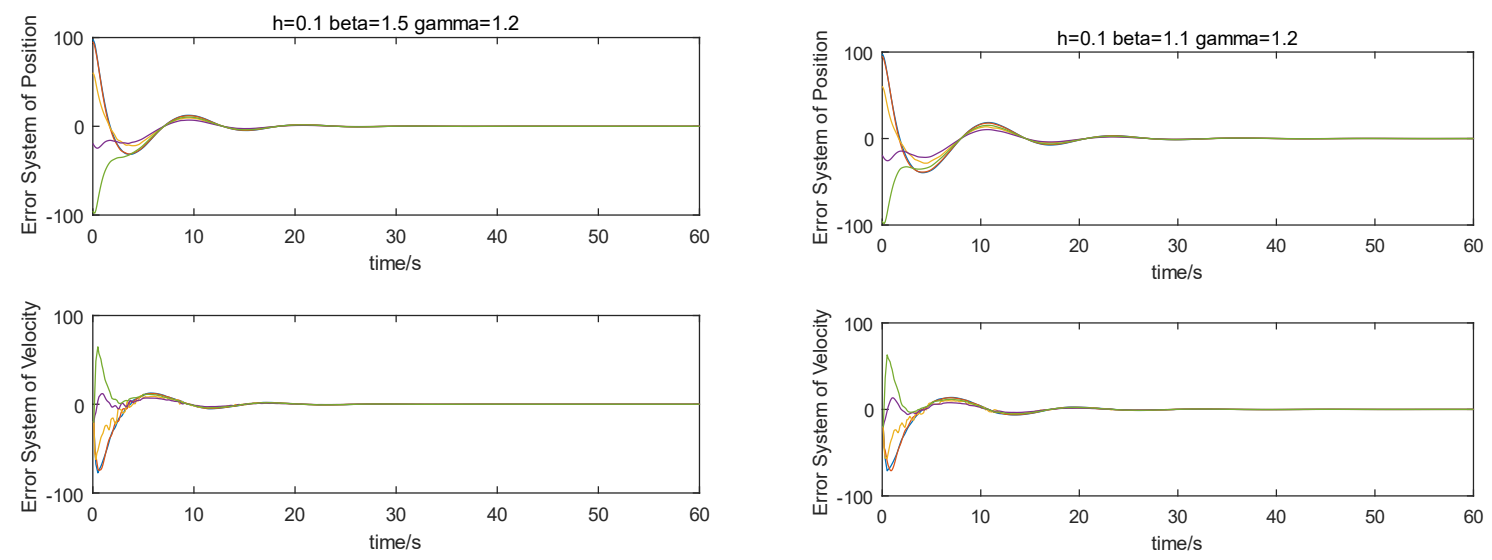

(a)
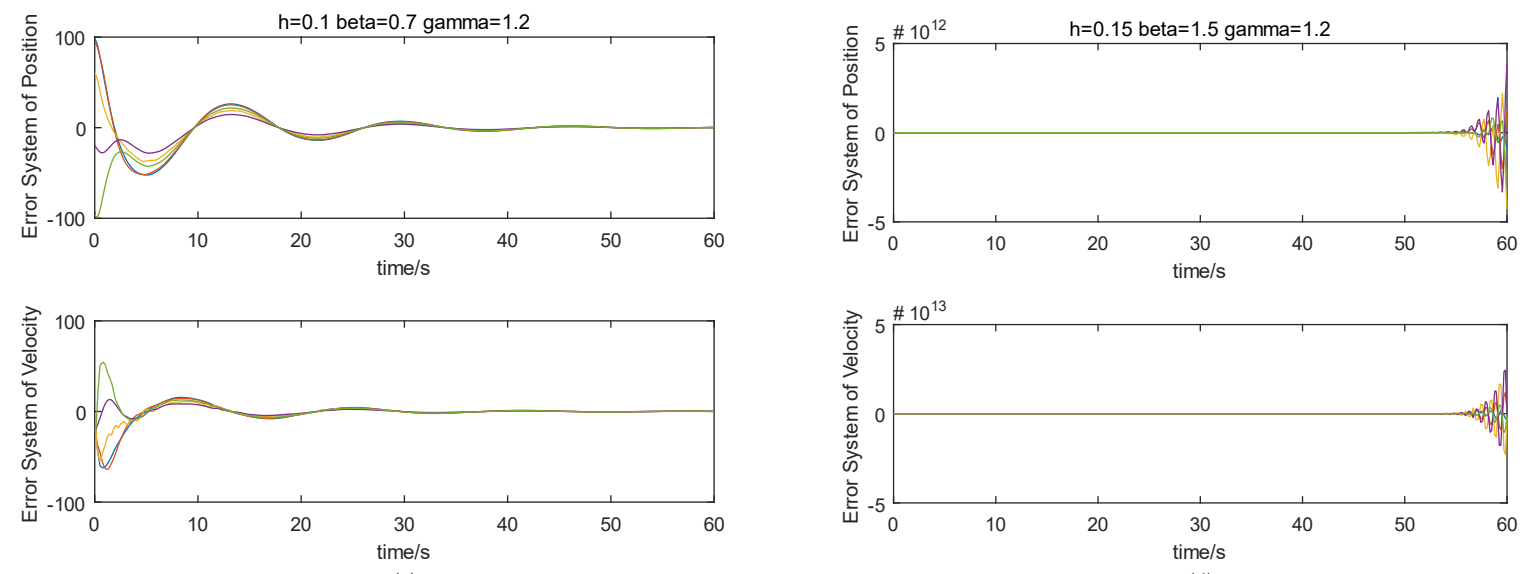

(d)

Fig. 2: Error system in experiment (a) - experiment(d). The setting of system parameters is shown in Table 1.

\section{Conclusions}

This paper illustrated the conditions to ensure the consensus of a system under distributed network when communication delay and switching topology existing. By constructing relevant models and then analyzing the system, we obtained the allowed bound of delay to guarantee consensus of the system. And finally, we provided simulation results to illustrate the correctness of our theorems. Our result provides a guide in both the design of controller and the optimizing of network protocol for consensus of the system under distributed network. Our works now are on the basis of time-invariant communication delay. We hope to analyze the influence of time-varying communication delay on consensus.

\section{Acknowledgements}

During the process of research, my tutor gave me lots of valuable advices, which helped me finish my study. My colleagues, Jing Zhang and Gangyang Li also encouraged me to do the research.

\section{References}

[1] LIU Chan, ZHU Yong-chuan, BAI Yuan, et,al. Flocking for Multi-Agent Clustering and Obstacle Avoidance: Algorithm and Simulation. Communications Technology. 2019, 52(07): 1632-1638. (in Chinese) 
[2] D. Sakai, H. Fukushima and F. Matsuno. Flocking for Multirobots Without Distinguishing Robots and Obstacles. IEEE Transactions on Control Systems Technology. 2017, 25(3): 1019-1027.

[3] AI Li. Distributed Unmanned Aerial Vehicles Formation Control with Nonlinear Dynamics. Journal of Chongqing University of Technology. 2020, 34(4): 170-175. (in Chinese)

[4] WU Y, LIANG T J. An improved consensus-based algorithm for unmanned aerial vehicle (UAV) formation control. Acta Aeronautica et Astronautica Sinica. 2020, 41(10): 1-18. (in Chinese)

[5] Zhou S L, Wang S L, Liu W, et al. Cooperative attitude control in rigid body swarms based on group consensus. Sci Sin Tech. 2020, 50(5): 493-505. (in Chinese)

[6] C. Yong, X. Guangming and L. Huiyang. Reaching consensus at a preset time: Single-integrator dynamics case. Proceedings of the 31st Chinese Control Conference, Hefei. 2012, pp. 6220-6225.

[7] W. Ren, R. W. Beard. Consensus seeking in multiagent systems under dynamically changing interaction topologies. IEEE Transactions on Automatic Control. 2005, 50(5): 655-661.

[8] X. Xu, L. Liu and G. Feng. Consensus of single integrator multi-agent systems with unbounded transmission delays. 2017 13th IEEE International Conference on Control \& Automation (ICCA). 2017, pp. 554-558.

[9] W. Ren, Y. Cao: Convergence of sampled-data consensus algorithms for double-integrator dynamics. 2008 47th IEEE Conference on Decision and Control. 2008, pp. 3965-3970.

[10] HE L L, ZHANG J Q, HOU Y Q, et al. Time-varying formation control for UAV swarm with directed interaction topology and communication delay. Journal of Beijing University of Aeronautics and Astronautics. 2020, 46(2): 314-323. (in Chinese)

[11] M. Xing, F. Deng, Z. Hu. Sampled-Data Consensus for Multiagent Systems with Time Delays and Packet Losses. IEEE Transactions on Systems, Man, and Cybernetics: Systems. 2020, 50(1): 203-210.

[12] S. Kar, J. M. F. Moura. Distributed Consensus Algorithms in Sensor Networks with Imperfect Communication: Link Failures and Channel Noise. IEEE Transactions on Signal Processing. 2009, 57(1): 355-369.

[13] Z. Wang, H. Zhang. Consensus Error Calculation for Multiagent Systems with Both System and Measurement Noises. IEEE Transactions on Control of Network Systems. 2018, 5(3): 1457-1466.

[14] The Homepage. https://en.wikipedia.org/wiki/Cubic_equation. 2020. 